\title{
Transplant Glomerulopathy: Importance of Ultrastructural Examination
}

\author{
Ian W. Gibson \\ Department of Pathology, University of Manitoba, Winnipeg, MB, Canada
}

\section{Key Points}

- Transplant glomerulopathy (TG) is a morphologic pattern reflecting chronic glomerular endothelial injury in renal allografts, characterized by remodeling and reduplications of the glomerular basement membrane (GBM).

- TG is a feature of chronic antibody-mediated injury, but other causes of GBM remodeling, including chronic thrombotic microangiopathy, produce similar morphologic appearances.

- The histologic diagnosis of TG is associated with poor graft prognosis and chronic progression to graft failure.

- Electron microscopic examination can identify early features of TG (Banff score cgla), including glomerular endothelial abnormalities, subendothelial widening, and early GBM reduplications.

- Routine ultrastructural examination is recommended in renal allograft biopsies, especially those at high risk of antibody-mediated rejection, for early diagnosis of TG when therapeutic interventions may be beneficial.

\section{Keywords}

Transplant glomerulopathy · Chronic antibody-mediated rejection · Renal allograft pathology

\begin{abstract}
Background: Transplant glomerulopathy (TG) is a morphologic alteration in glomeruli of renal allografts, characterized by glomerular basement membrane reduplications. Summary: TG is associated with progressive chronic allograft dysfunction and proteinuria and is a diagnostic feature of chronic antibody-mediated rejection (ABMR) in patients positive for donor-specific antibodies, according to the Banff schema for renal allograft pathology. It is a definitive endpoint in clinical trials and interventional studies for ABMR, but the lesion can also occur in the absence of definitive al-
\end{abstract}

loimmune injury, as a consequence of chronic thrombotic microangiopathy, and in some cases in association with hepatitis $C$ infection. This review discusses the pathophysiology and clinical presentation of TG, the diagnostic features by light microscopy, and focuses on the sequential ultrastructural stages of the lesion. The differential diagnosis of TG, and Banff grading of the lesion, are reviewed. Clinicopathological indications for performing routine ultrastructural examination of renal allograft biopsies are discussed. Key Messages: TG can be diagnosed at an early stage by electron microscopy, before histological features are apparent, emphasizing the importance of ultrastructural examination of renal allograft biopsies for an early diagnosis, when therapeutic intervention may be beneficial.

(C) 2021 The Author(s)

Published by S. Karger AG, Basel karger@karger.com www.karger.com/gdz

Karger $\stackrel{\text { ' }}{5}$

BOPEN ACCESS
(C) 2021 The Author(s)

Published by S. Karger AG, Basel

This article is licensed under the Creative Commons AttributionNonCommercial-NoDerivatives 4.0 International License (CC BYNC-ND) (http://www.karger.com/Services/OpenAccessLicense) Usage and distribution for commercial purposes as well as any distribution of modified material requires written permission.
Ian W. Gibson

Department of Pathology, MS-336C Electron Microscopy Lab, Health Sciences Centre University of Manitoba

820 Sherbrook Street, Winnipeg, MB R3A1R9 (Canada)

IGibson@sharedhealthmb.ca 


\section{Introduction}

Transplant glomerulopathy (TG) is not a specific diagnosis but is a morphologic pattern of chronic glomerular injury in renal allografts characterized by remodeling and reduplications of the glomerular basement membrane $(\mathrm{GBM})$. The prevalence of TG is approximately $5-10 \%$ of renal allografts at 5 years post-transplantation; rarely it can be a subclinical finding in protocol biopsies. The lesion is associated particularly with chronic alloantibody-mediated injury and generally has a poor prognosis for graft outcome.

\section{Pathophysiology}

TG is a chronic slowly progressive injury to glomerular capillary walls, presumed to be the consequence of chronic glomerular endothelial activation and injury, with associated repair and remodeling of the GBM [1]. Glomerular epithelial cell stress and reduced podocyte density also play a role in transplant-related glomerular injury, including significant podocyte detachment and increased urinary podocyte excretion in TG [2], with associated progressive glomerulosclerosis.

In antibody-mediated rejection (ABMR), sub-lethal complement-mediated chronic endothelial injury is associated with circulating donor-specific anti-HLA antibodies (DSA), either class I or more commonly class II, interacting with HLA antigens expressed by the glomerular endothelium, and possibly also expressed by podocytes. Approximately 75\% of allografts with TG have associated concurrent or prior DSA, consistent with chronic ABMR [1]. Molecular studies of allografts with alloantibody-mediated TG $[3,4]$ show increased expression of endothelial gene transcripts, such as Duffy blood group antigen, von Willibrand factor, adhesion molecules MCAM and ICAM-1, and gamma interferon, consistent with chronic endothelial activation and injury.

In pre-sensitized positive crossmatch patients with pre-existing DSA, early ultrastructural lesions of TG can be found as early as 3 months post-transplant and can progress to established TG within 1 year post-transplant in approximately $20 \%$ of cases [5]. In patients who develop de novo DSA, the progression to overt TG is generally more protracted over many years [6]. ABO-incompatible renal allografts with DSA against blood group $\mathrm{ABO}$ antigens have a mildly increased risk of TG [7]. Non-HLA antibody-antigen interactions can also be associated with development of TG [8], including post-transplant autoan-

Transplant Glomerulopathy tibodies to the GBM protein agrin [9] and to the ubiquitous intermediate filament vimentin [10].

Transplant glomerulitis can be a pre-existing lesion of TG, with an intermediate chronic active stage of ABMR showing both lesions [11]. Animal models of ABMR have demonstrated morphologic progression from active glomerulitis to the chronic lesion of TG [12], and serial protocol biopsy studies in human ABMR suggest a similar temporal progression in both pre-sensitized patients [5] and with development of de novo DSA [13]. The evidence for chronic T-cell-mediated rejection (TCMR) as a cause for TG is less definitive, but given that TCMR can be a cause for transplant glomerulitis, the possibility of progression to TG cannot be discounted.

Chronic hepatitis $C$ virus infection is also a recognized association of TG in several studies [14-16], not associated with circulating cryoglobulins or glomerular immune complex deposition [17]. HCV-positive TG has a worse graft outcome compared with HCV-negative TG [16], possibly related to antiphospholipid antibody-mediated thrombotic microangiopathy. There are thus overlapping pathways to development of TG, for example, any cause of chronic glomerular thrombotic microangiopathy, including chronic calcineurin inhibitor nephrotoxicity, can result in a morphologic lesion identical to TG.

Several animal models of TG have been developed. A rat model used Fisher-344 kidneys transplanted into Lewis rats without immunosuppression [18]. Graft harvesting at sequential time points up to 26 weeks showed glomerular lesions typical of human acute and chronic $\mathrm{ABMR}$, including glomerular hypertrophy, glomerulitis, microthrombi, microaneurysms, loss of podocytes, GBM reduplications, and secondary focal and segmental glomerulosclerosis. Glomerular subendothelial and mesangial electron dense immune complex-type deposits were also identified in this rodent model at 26 weeks posttransplant, comparable to a subset of human TG biopsies, raising the possibility of alloantibodies directed against non-MHC GBM or mesangial epitopes, and in situ immune complex deposition in the pathogenesis of TG.

A nonhuman primate model of chronic ABMR in renal allografts in cynomolgus monkeys has also been extensively studied [12] and shows similar progression of renal pathology lesions analogous to human allografts, with microvascular inflammation, C4d deposition, and progression to TG and chronic arteriopathy. Molecular analysis for gene transcripts related to ABMR showed similar findings in this primate model to human $A B M R$, with several endothelial genes (VWF, DARC, and CAV1) being discriminatory for ABMR [19]. 

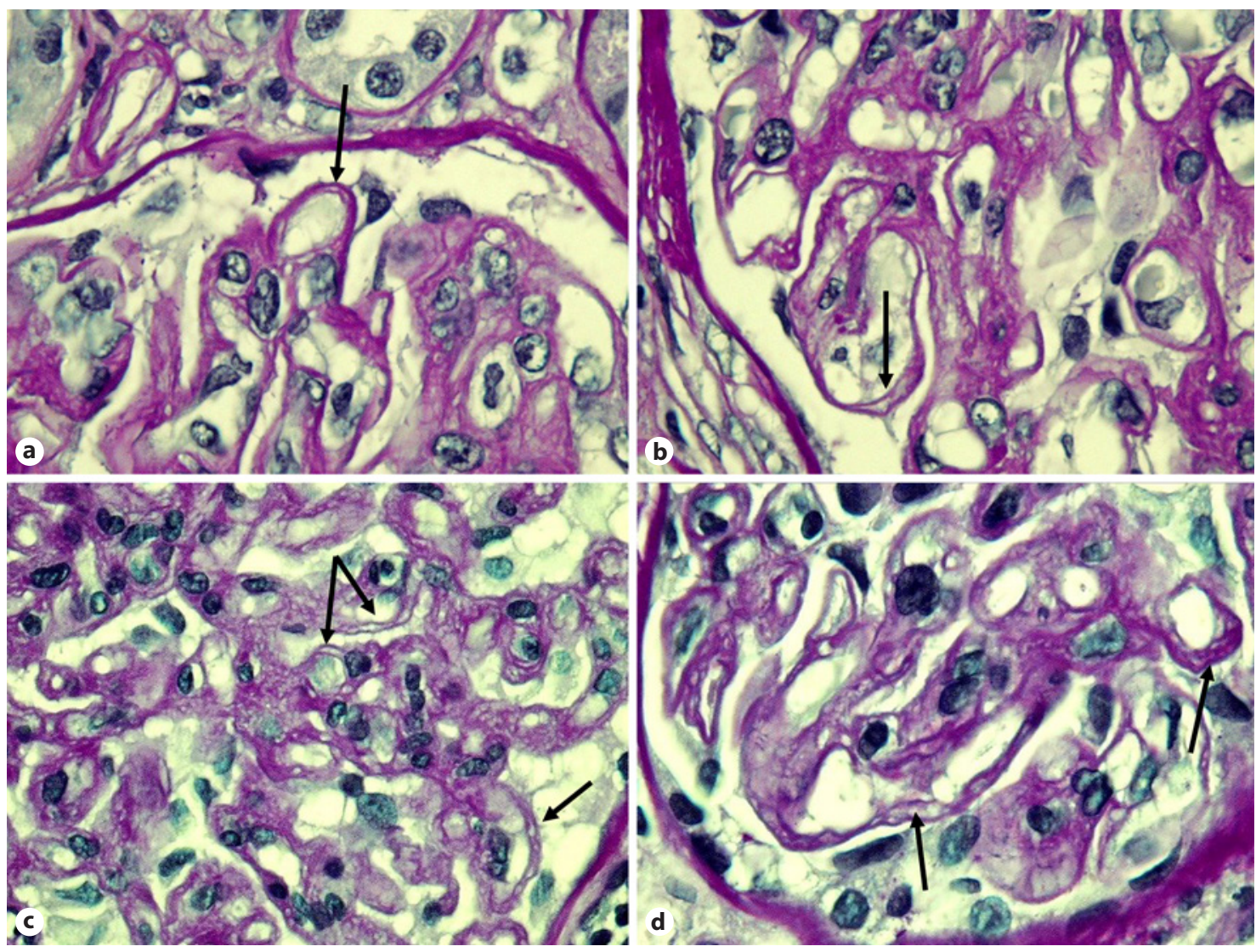

Fig. 1. Highly sensitized patient, 2nd renal allograft, with multiple preformed HLA class I and II DSAs (A1, A32, Cw9, DP3, DP17). Earlier allograft biopsies showed C4d-positive active ABMR and TCMR treated aggressively with plasmapheresis, thymoglobulin, and OKT3. Developed proteinuria and progressive graft dysfunction, with rapidly evolving TG due to ABMR, progressing to graft failure at 20 months post-transplant. a, b Renal allograft biopsy at 4.5 months post-transplant with TG (Banff score cg1). There are

\section{Clinical Manifestations}

TG can be a subclinical finding in protocol allograft biopsies from patients with DSA, with higher risk associated with HLA class II DSA [20]. Once TG presents clinically, there is usually proteinuria of sub-nephrotic or nephrotic range. As the lesion progresses, there is indolent graft dysfunction with slowly rising serum Cr levels, hypertension, and shortened graft survival. There can be a disconnect between the severity of clinical and pathological features, with a relatively low level of proteinuria, but an established TG lesion identified ultrastructurally.

Once TG is demonstrated histologically on renal allograft biopsy, the prognosis for graft survival is poor, with progressive graft dysfunction and increasing pro- scattered glomerular capillaries with GBM reduplications (arrows). PAS stain, $\times 600$. c, d Renal allograft biopsy at 11 months post-transplant with severe TG (Banff score cg3), with glomerular capillaries showing multiple irregular GBM reduplications (arrows). PAS stain, $\times 600$. TG, transplant glomerulopathy; GBM, glomerular basement membrane; ABMR, antibody-mediated rejection; DSA, donor-specific antibody; TCMR, T-cell-mediated rejection.

teinuria. In a series of crossmatch negative kidney recipients, graft survival was $62 \%$ at 5 years post-transplantation in patients with TG, compared with $95 \%$ in those without TG [21]. Some studies have found that the prognosis for TG is worse when there is associated active microvascular inflammation [4], and when C4d staining is positive in peritubular capillaries [22].

A recent large multicenter study of TG [23] showed that by integrating clinical, functional, immunologic and histologic data, 5 distinct groups of TG could be identified, with differences in allograft survival at 5 years after diagnosis of TG ranging from 22 to $88 \%$. The worst prognosis group presented later post-transplant, with higher levels of proteinuria, and higher Banff scores for chronic tubulointerstitial and vascular damage. A significant 


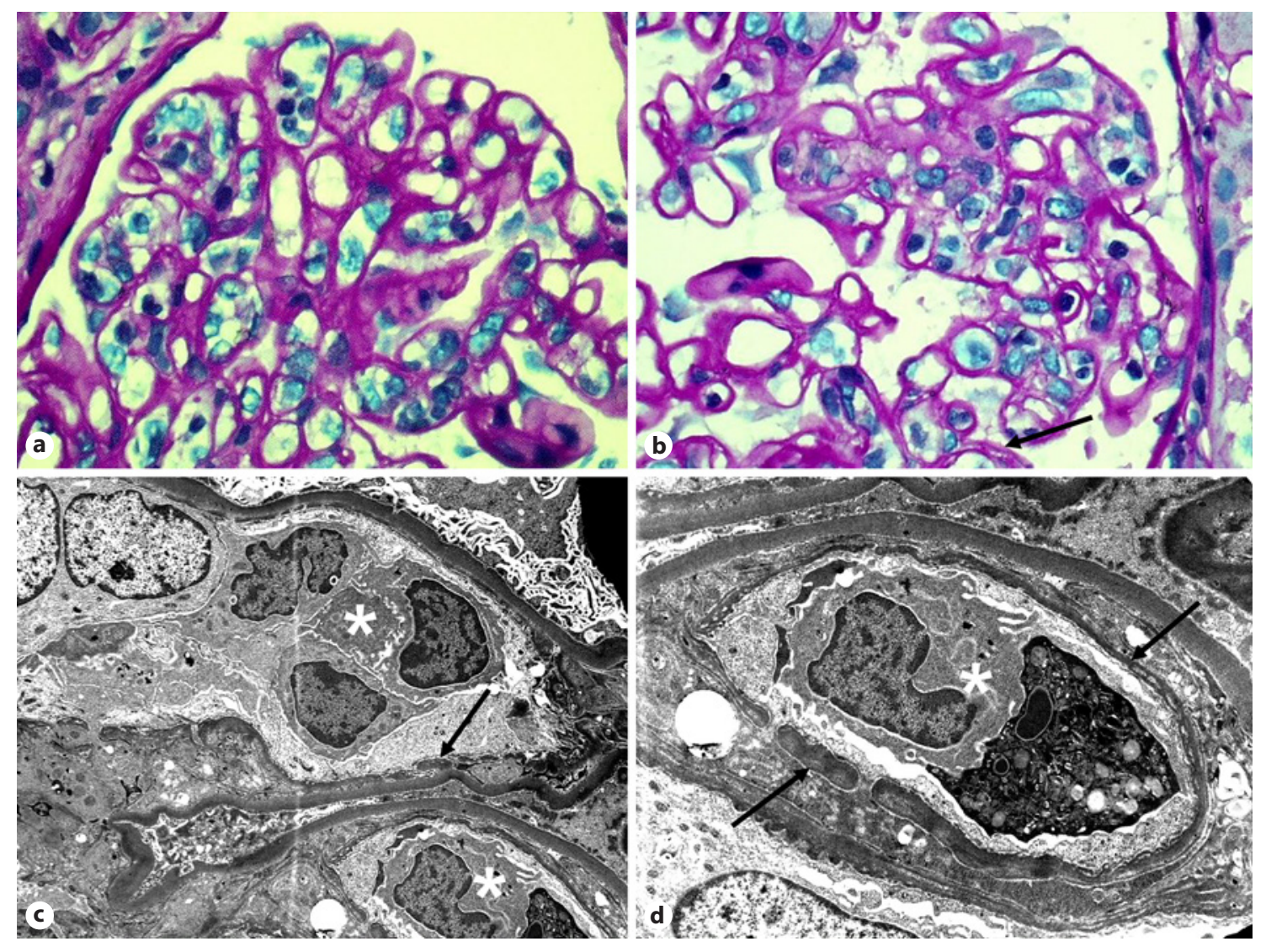

Fig. 2. Renal allograft biopsy at 11 years post-transplant; patient has de novo HLA class I (A1) DSA since 8 years post-transplant. There is active glomerulitis (Banff score g2) with prominent endothelial swelling and endocapillary mononuclear inflammatory cells $(\mathbf{a}, \mathbf{b})$, forming endocapillary occlusive lesions $\left(\mathbf{c}, \mathbf{d}^{*}\right)$. There is established chronic TG (Banff score cg1) with GBM reduplica- tions by LM (b arrow), and subendothelial widening with focal circumferential GBM reduplication (c, d arrows) by EM. a, b PAS stain, $\times 600$. $\mathbf{c} \times 6,000$. $\mathbf{d} \times 10,000$. TG, transplant glomerulopathy; GBM, glomerular basement membrane; DSA, donor-specific antibody; LM, light microscopy; EM, electron microscopy. number of TG cases in this study showed a mixed rejection phenotype, with lesions of TCMR as well as chronic active ABMR. A recently published literature review and meta-analysis [24], incorporating a total of 21 studies and 6,783 patients, showed that TG is associated with a 3 -fold increase in risk of graft failure, and a 15 -year shorter graft survival.

There is no good evidence that biopsy-proven TG identified by light microscopy (LM) can be reversed or that decline in graft function can be significantly delayed, with the presently available treatments for chronic ABMR [25]. However, if TG is diagnosed at an early ultrastructural stage (Banff cgla) in protocol biopsies at 3 months post-transplant, aggressive treatment for ABMR with high-dose IVIG, plasmapheresis, and/or rituximab may prevent further progression of the lesion to overt TG in some cases [26]. Some investigators have found that treatment of established TG with rituximab and IVIG may help stabilize level of proteinuria and preserve graft function [27]. Also treatment with tocilizumab (anti-IL6 monoclonal antibody) has been reported to stabilize graft function in patients with chronic active ABMR, although biopsy-proven TG scores remained stable after such treatment $[28,29]$.

\section{Light Microscopy}

TG is identified histologically by reduplications or "double contours" of the GBM in peripheral glomerular capillary loops (Fig. 1a-d, 2a, b), best identified with basement membrane stains (periodic acid-Schiff and methanamine silver). In early stages, it is a focal lesion present 

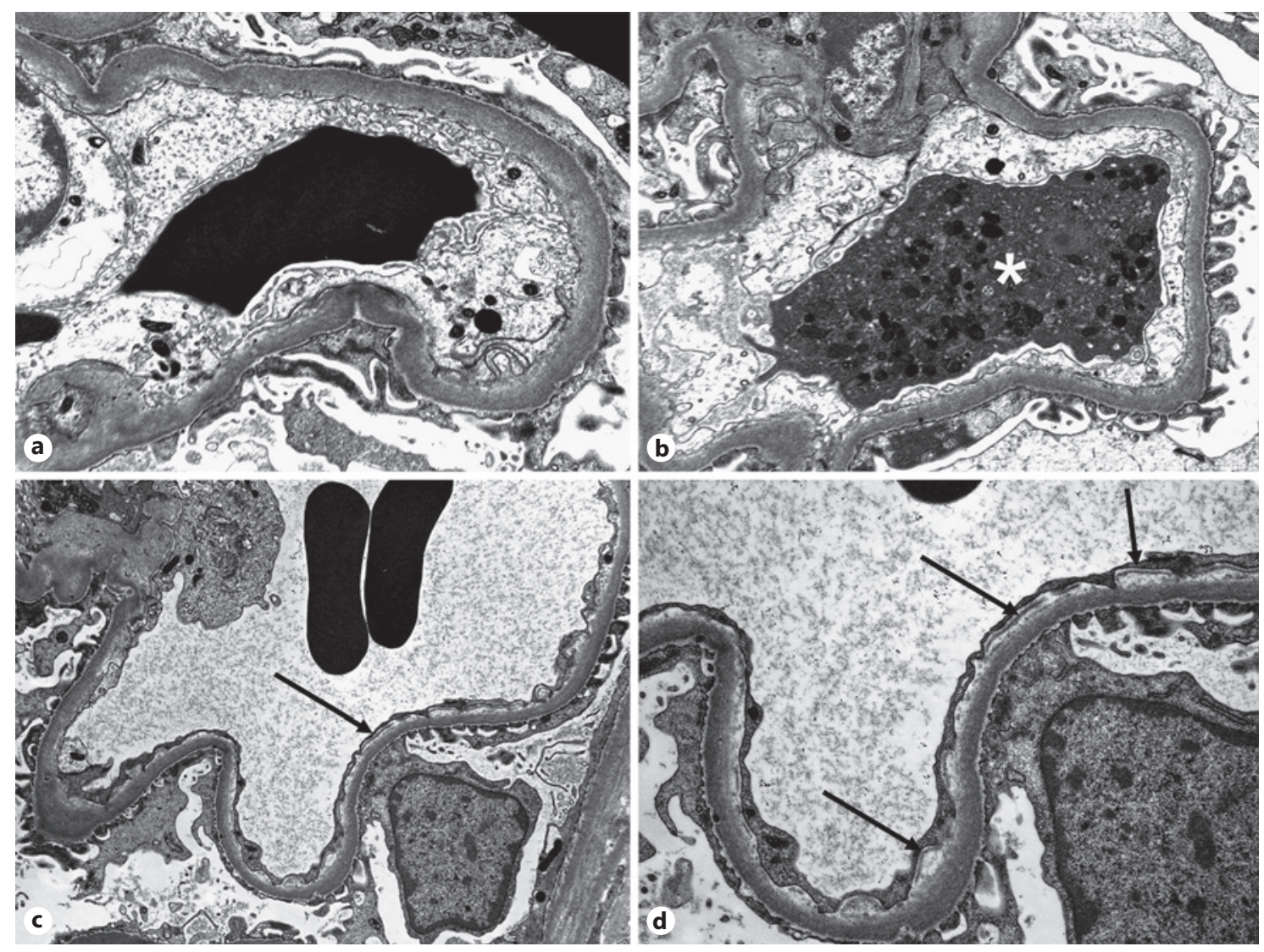

Fig. 3. Protocol renal allograft biopsy at 1 year post-transplant. Prolonged delayed graft function after transplantation, with previous biopsies in the first month post-transplant showing acute tubular necrosis and early vascular rejection (Banff grade IIB). No significant proteinuria and negative for DSA at time of this biopsy. Glomerular capillaries show endothelial cell activation and swelling, with loss of normal fenestrations $(\mathbf{a}, \mathbf{b})$, and luminal inflam- matory cell $\left(\mathbf{b}^{*}\right)$. There is very early TG (Banff score cg1a) with focal subendothelial widening and incomplete thin GBM reduplication (c, $\mathbf{d}$ arrows) identified in occasional glomerular capillaries. a $\times 15,000$. b $\times 15,000$. $\mathbf{c} \times 10,000$. d $\times 20,000$. TG, transplant glomerulopathy; GBM, glomerular basement membrane; DSA, donor-specific antibody. in a minority of glomeruli, so adequate glomerular sampling is necessary. As the lesion progresses, it becomes more diffuse, with associated glomerular hypertrophy, variable mesangial matrix expansion (without mesangial nodule formation or significant mesangial cell proliferation), and secondary focal segmental and global glomerulosclerosis and hyalinosis. Advanced TG would usually be associated with progressive interstitial fibrosis and tubular atrophy, arterial fibrous intimal thickening, and severe nodular hyaline arteriolosclerosis.

TG associated with DSA is often seen in association with some degree of active microvascular inflammation, specifically transplant glomerulitis and peritubular capillaritis. Transplant glomerulitis is characterized by intracapillary aggregation and margination of mononuclear leukocytes and/or neutrophils, and endothelial cell swell- ing, causing variable capillary luminal occlusion (Fig. 2ad). In the presence of documented DSA, the combination of active glomerulitis and peritubular capillaritis with TG would be diagnostic of chronic active ABMR.

\section{Immunofluorescence Microscopy}

Immunofluorescence (IF) findings in TG include mild-to-moderate glomerular mesangial and capillary wall deposition of IgM and C3, and no diagnostic staining for IgG, IgA, and C1q. Glomerular IF studies are most important for excluding the presence of a recurrent or de novo immune complex-mediated glomerulonephritis. A recent study found glomerular C3 deposition to be an independent risk factor for allograft failure in TG [30]. 


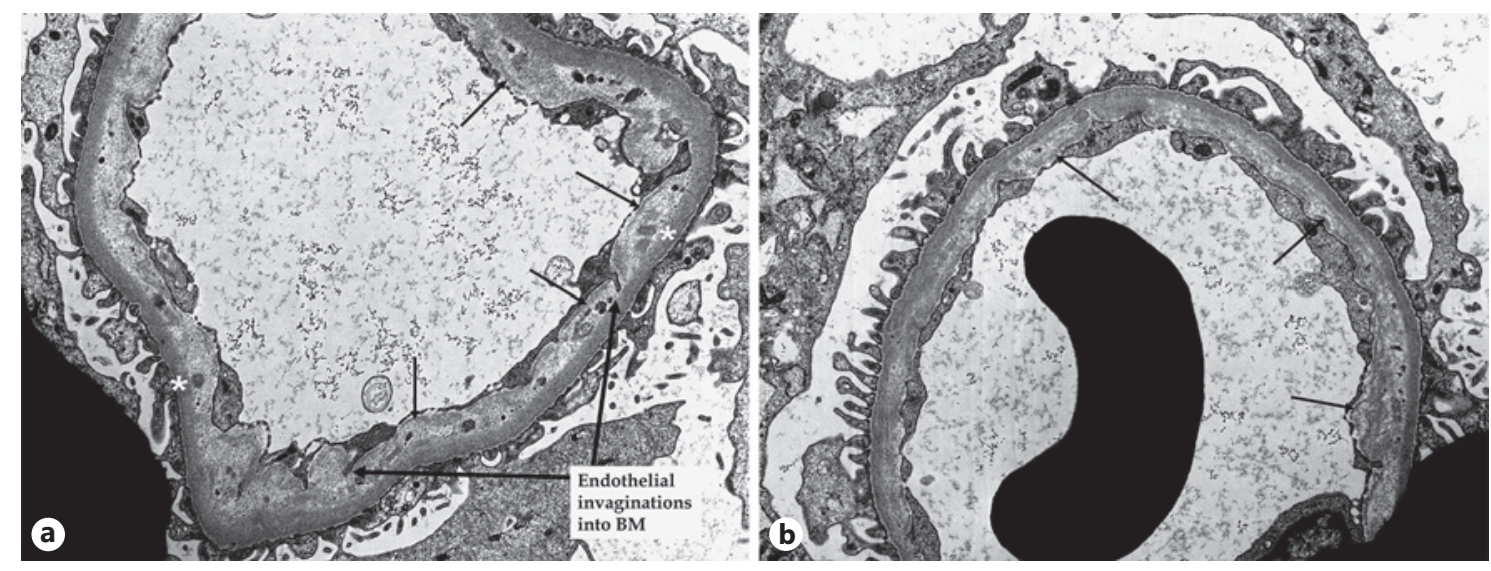

Fig. 4. Renal allograft biopsy at 6 years post-transplant; recently detected de novo HLA class II (DQ2) DSA. Glomerular capillaries showing early ultrastructural features of TG (Banff score cgla) with circumferential subendothelial widening, endothelial invaginations into GBM (a large arrows), and discontinuous thin GBM reduplications (a, b small arrows). Occasional small subendothelial electron dense deposits are also present $\left(\mathbf{a}^{*}\right)$. $\mathbf{a} \times 10,000$. $\mathbf{b} \times 10,000$. TG, transplant glomerulopathy; GBM, glomerular basement membrane; DSA, donor-specific antibody.

Staining for C4d (by IF or immunohistochemistry) is often positive in the glomerular capillary walls in TG, particularly when severe, with strong segmental or global pseudolinear, trans-membranous pattern of capillary loop staining [31], distinct from the nonspecific glomerular mesangial C4d staining. This glomerular capillary wall C4d deposition reflects structural GBM remodeling and can also be seen in various native and transplant-related glomerulopathies, including thrombotic microangiopathies and hereditary molecular GBM lesions [31, 32]. Thus, pseudolinear C4d staining along glomerular capillary walls, in particular when seen in isolation and without accompanying peritubular capillary staining in transplants, does not indicate a specific underlying disease etiology, such as antibody-induced injury. Glomerular C4d staining is not used to determine the $\mathrm{C} 4 \mathrm{~d}$ status of a renal allograft biopsy, which is based only on extent of peritubular capillary staining.

\section{Electron Microscopy}

In the earliest stage of TG (Fig. 3), the normal thin fenestrated glomerular endothelium shows an activated phenotype, characterized by cytoplasmic swelling, abundant mitochondria, enlarged Golgi apparatus, cytoplasmic vacuolization, loss of fenestrations producing a continuous cytoplasm, and an irregular serrated endothelial border along the GBM. Sequential ultra- structural studies in protocol and indication allograft biopsies show that these earliest morphologic features of evolving TG can be identified in the glomerular endothelium as early as 1 month post-transplantation [26, 33]. In pre-sensitized patients with pre-existing DSA, endothelial cell swelling can be seen as early as day 3 post-transplant [26].

These early glomerular endothelial cell abnormalities are associated with focal GBM abnormalities, specifically expansion of the lamina rara interna producing subendothelial electron-lucent widening, and very early basement membrane reduplication on the endothelial aspect of the GBM (Fig. 3c, d, 4a, b). The thin layer of reduplicated GBM is initially incomplete, and interrupted by invaginations of endothelial cell cytoplasm into the expanded subendothelial portion of the GBM (Fig. 4a, 6a). If these ultrastructural findings of early GBM reduplication are identified in at least 3 distinct glomerular capillary loops, in the absence of overt TG by LM, a diagnosis of Banff score cgla can be made, as recommended at the 2013 Banff conference [34]. The study by Haas and Mirocha [26] showed that the presence of all 3 early ultrastructural features of endothelial cell swelling, subendothelial widening, and early GBM reduplication was a consistent finding for evolving TG associated with ABMR.

With progression of TG (Fig. 5-7), the reduplicated subendothelial layer of GBM becomes circumferential around the affected capillary loop. Cellular interposition 

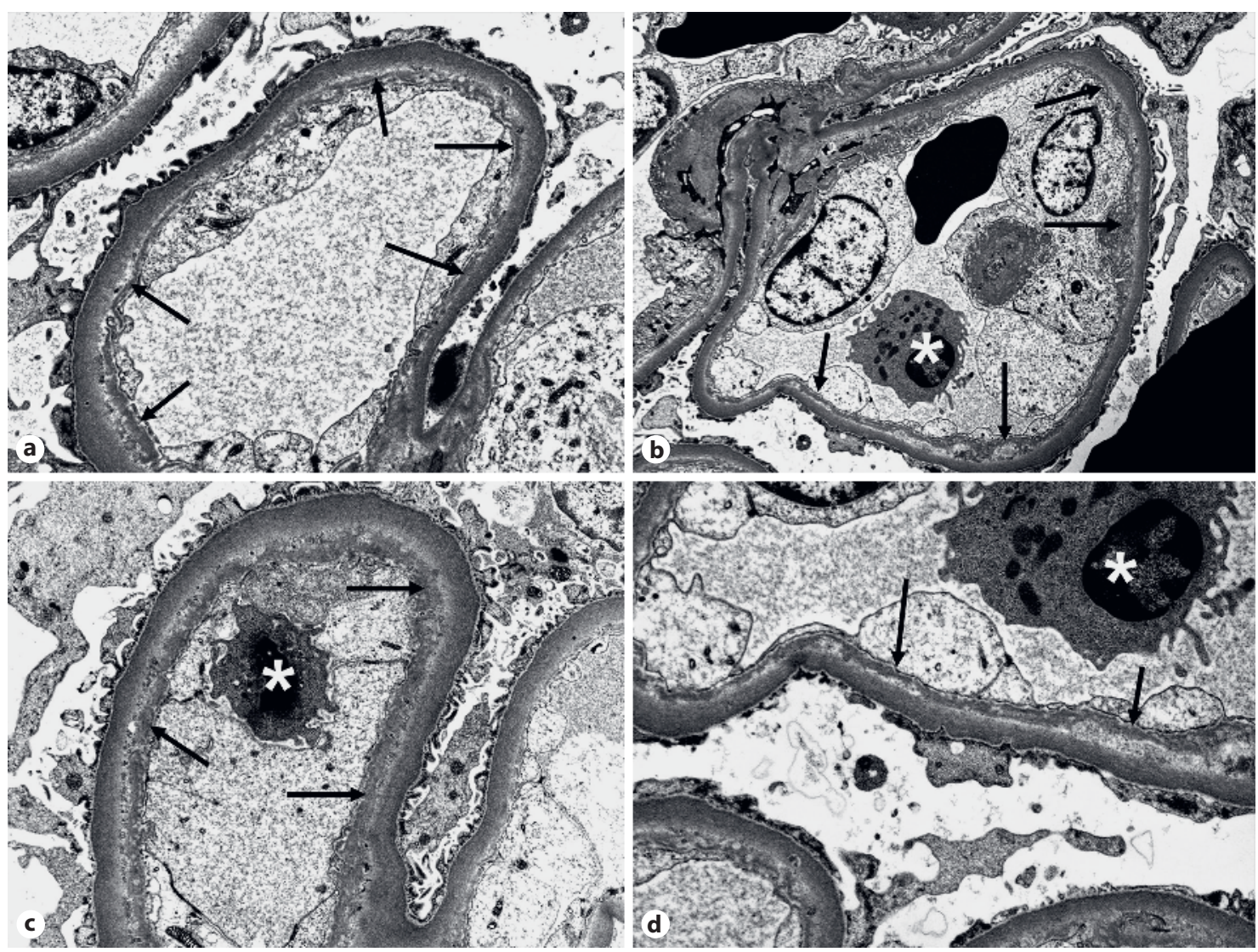

Fig. 5. Renal allograft biopsy at 9 years post-transplant; de novo HLA class II (DQ7). Glomerular capillaries with severe endothelial cell swelling, subendothelial widening, and continuous circumferential GBM reduplication of variable thickness (arrows). Luminal inflammatory cells are present in $\mathbf{b}-\mathbf{d}(*)$. $\mathbf{a} \times 12,000$. b $\times 6,000 . \mathbf{c} \times 10,000$. d $\times 15,000$. GBM, glomerular basement membrane.
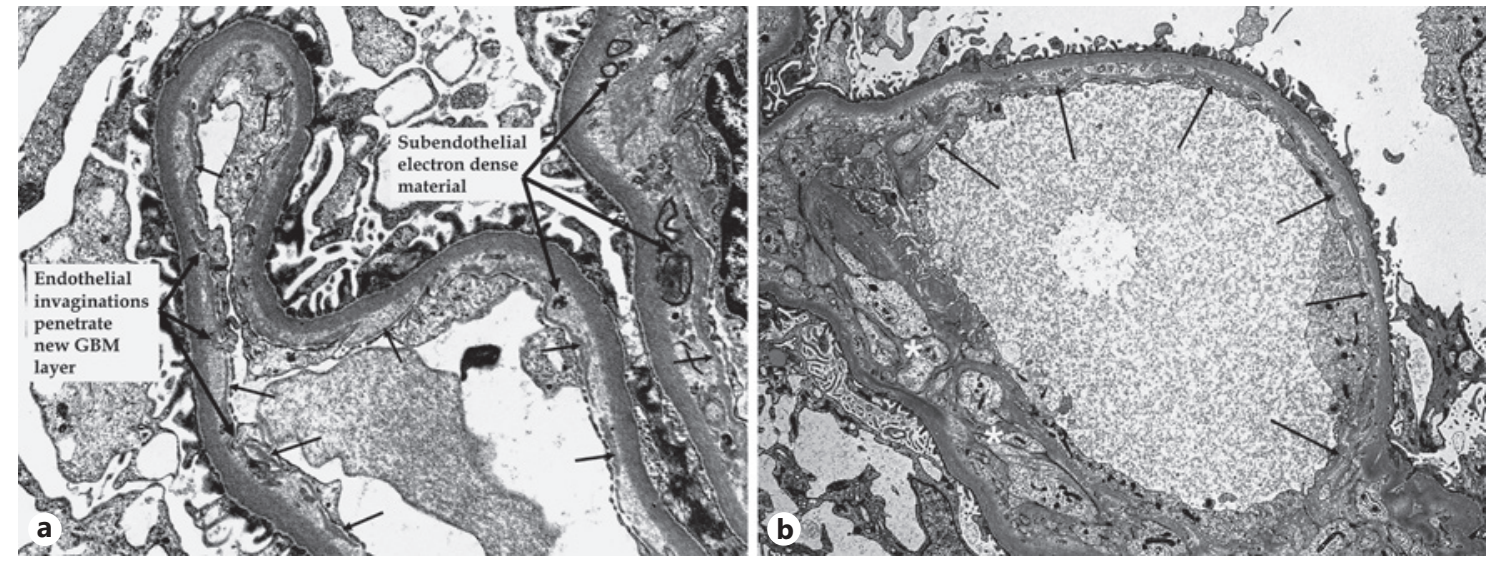

Fig. 6. Renal allograft biopsy at 17 years post-transplant; de novo HLA class I (A1) and II (DR53,DR13) DSA. Glomerular capillaries with subendothelial widening, discontinuous (a) and circumferential continuous (b) GBM reduplication (small arrows), endothelial invaginations penetrate the new GBM layer (a large arrows), scattered subendothelial nonspecific electron dense material (a large arrows), and cellular interposition (b *) into expanded subendothelial space. $\mathbf{a} \times 12,000$. $\mathbf{b} \times 4,000$. GBM, glomerular basement membrane; DSA, donor-specific antibody. 

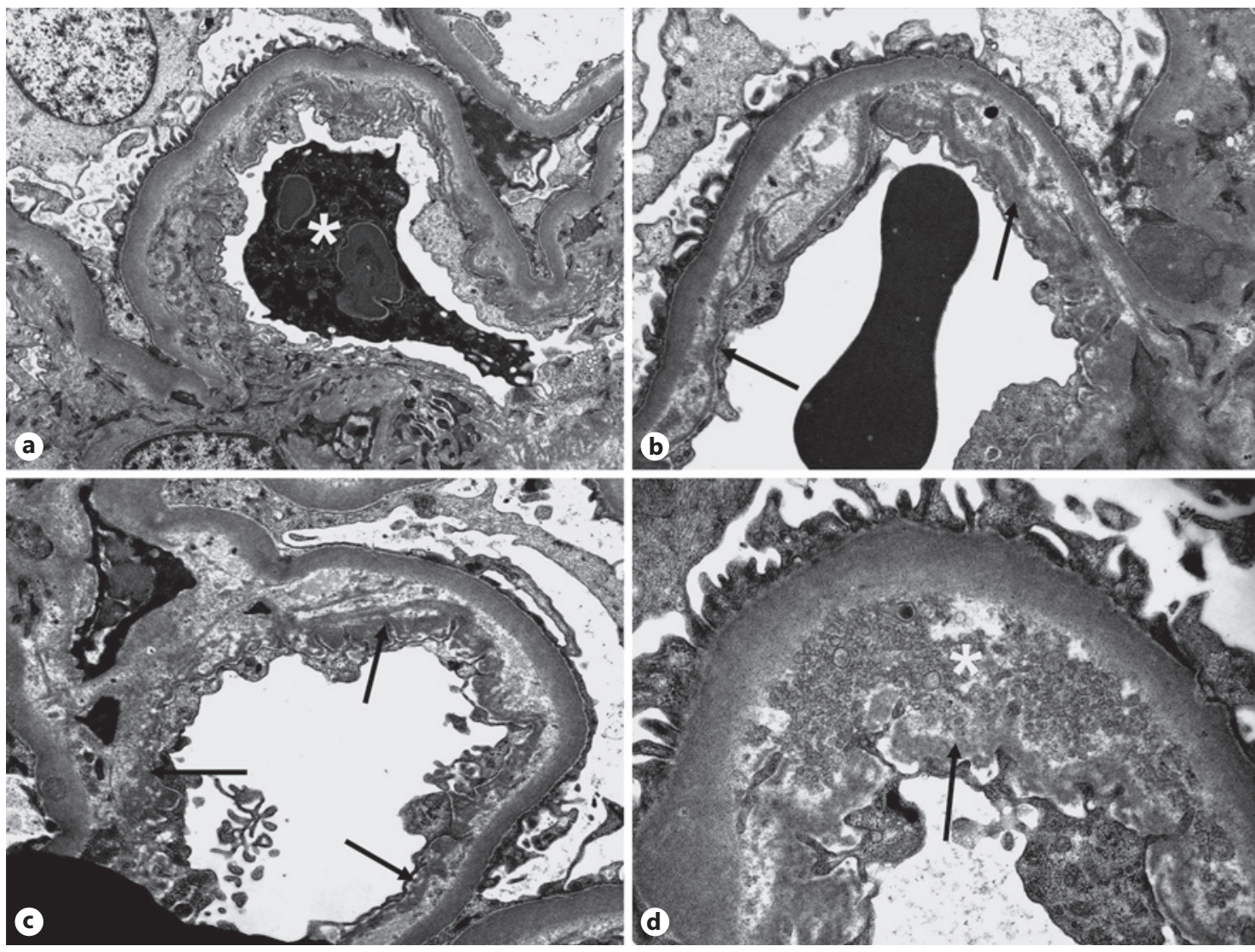

Fig. 7. Renal allograft biopsy at 13 years post-transplant; de novo HLA class I (B13) DSA since 11 years posttransplant; $<1 \mathrm{~g} /$ day proteinuria, serum $\mathrm{Cr} 128 \mu \mathrm{mol} / \mathrm{L}$. LM showed extensive GBM reduplications (Banff score cg3). Glomerular capillaries with an intraluminal neutrophil $\left(\mathbf{a}^{*}\right)$, continuous circumferential GBM reduplication (b, $\mathbf{c}$ arrows), and subendothelial widening with focally prominent heterogeneous nonspecific electron dense material $\left(\mathbf{d}^{*}\right)$. $\mathbf{a} \times 8,000$. b $\times 15,000$. $\mathbf{c} \times 12,000$. $\mathbf{d} \times 25,000$. GBM, glomerular basement membrane; DSA, donorspecific antibody; LM, light microscopy.

Fig. 8. Renal allograft biopsy at 14 years post-transplant; de novo HLA class I (A11) and II (DQ9) DSA; 6 g/day proteinuria. Glomerular capillaries with multiple circumferential GBM reduplications (small arrows) and cellular interposition in the widened subendothelial space (large arrow). $\times 10,000$. GBM, glomerular basement membrane; DSA, donor-specific antibody.

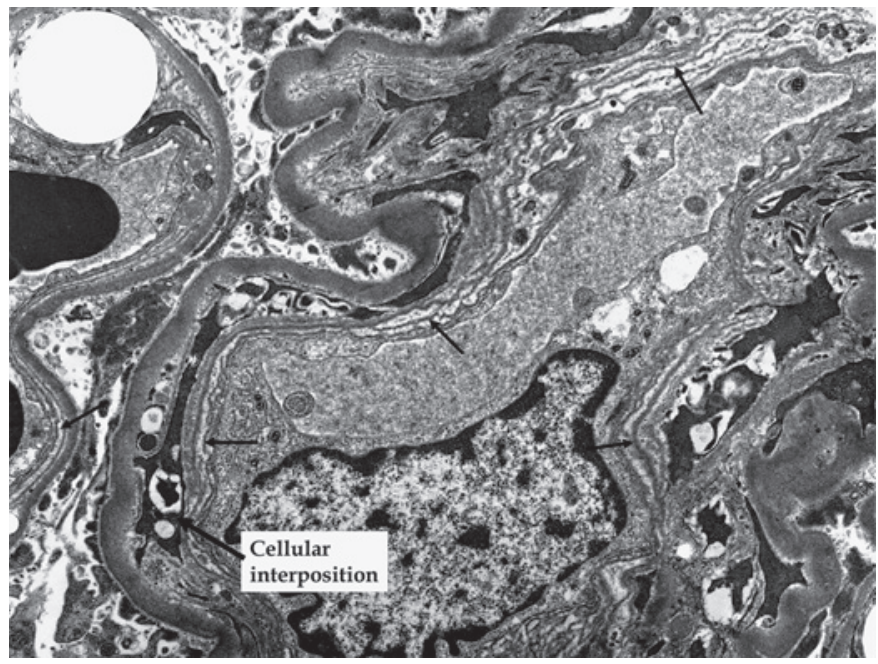




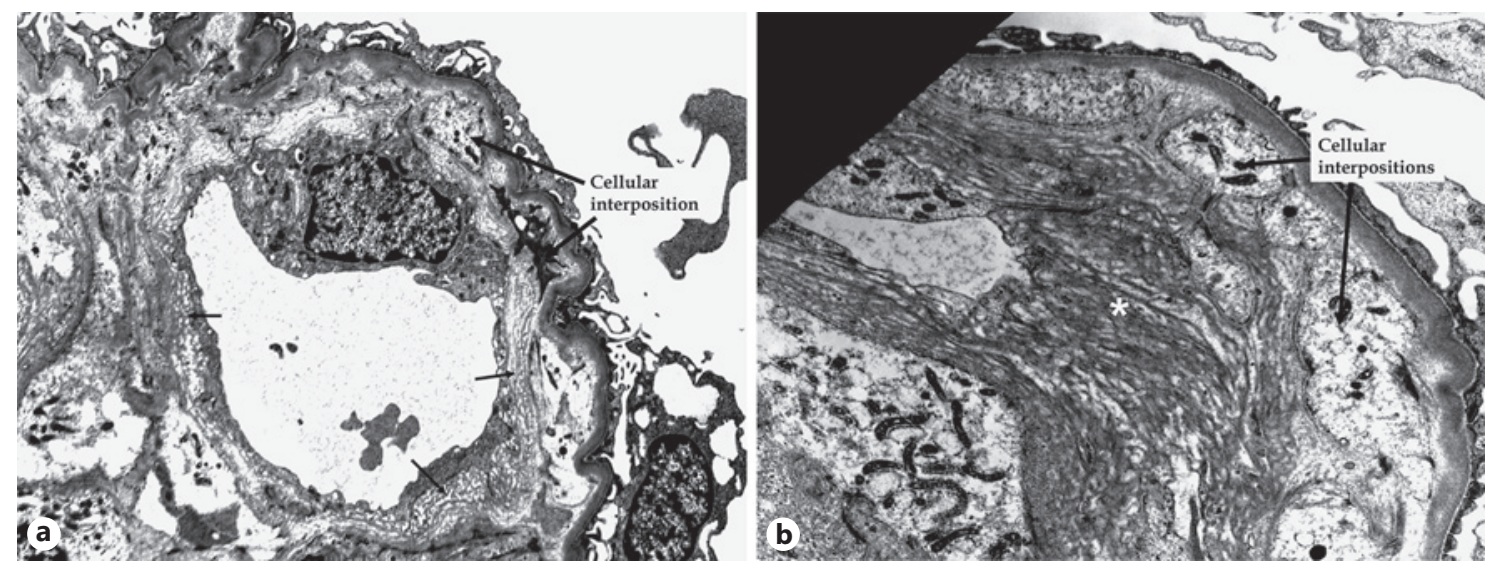

Fig. 9. Renal allograft biopsy at 6 years post-transplant; de novo HLA class I (A1, A68) DSA; $2.4 \mathrm{~g} /$ day proteinuria, serum Cr $188 \mu \mathrm{mol} / \mathrm{L}$. Glomerular capillary with multiple circumferential GBM reduplications (a small arrows), forming an interconnecting meshwork of base- ment membrane layers $\left(\mathbf{b}^{*}\right)$. There are cellular interpositions in the widened subendothelial space (a, b large arrows), and patchy glomerular epithelial cell foot process effacement. $\mathbf{a} \times 6,000$. $\mathbf{b} \times 10,000$. GBM, glomerular basement membrane; DSA, donor-specific antibody.
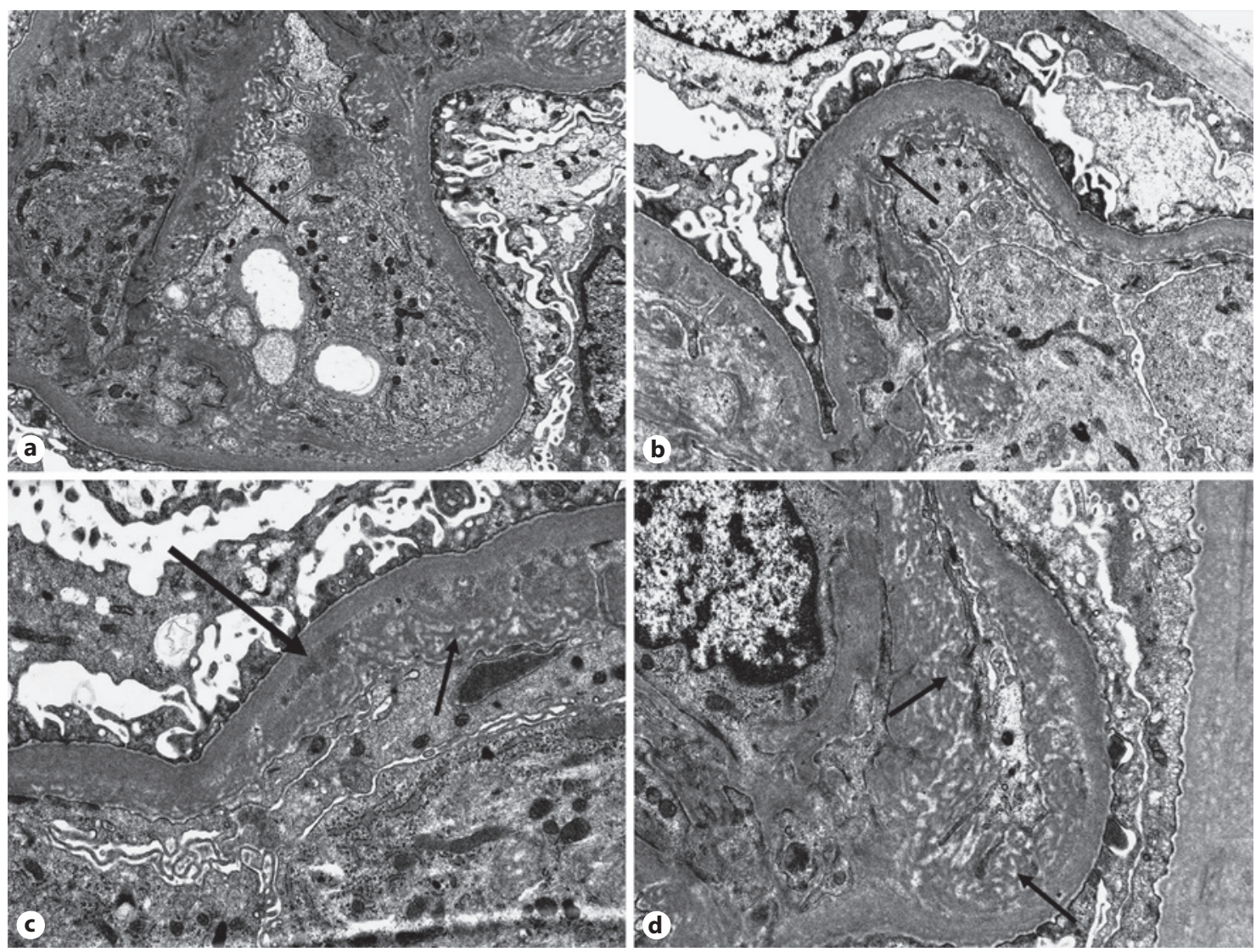

Fig. 10. Renal allograft biopsy at 7 years post-transplant; de novo HLA class II (DQ4) DSA since 1 year post-transplant; 4 g/day proteinuria, serum $\mathrm{Cr}>400 \mu \mathrm{mol} / \mathrm{L}$, GFR $<20 \mathrm{~mL} / \mathrm{min}$. LM showed severe TG (Banff score cg3) with extensive global glomerulosclerosis (>50\% of glomeruli) and severe scarring with approximately $70 \%$ cortical IFTA. IF showed patchy glomerular capillary loop (1-2+) IgM and C4d; negative for IgG, IgA, C3, and C1q. Patient progressed rapidly to graft failure. Glomerular capillaries showing endothelial cell swelling, subendothelial widening with scattered electron dense deposits (c large arrow), and multiple irregular GBM reduplications (small arrows). a $\times 10,000$. b $\times 12,000$. $\mathbf{c} \times 15,000 . \mathbf{d} \times 15,000$. TG, transplant glomerulopathy; GBM, glomerular basement membrane; DSA, donor-specific antibody; IFTA, interstitial fibrosis and tubular atrophy; IF, immunofluorescence; LM, light microscopy. 


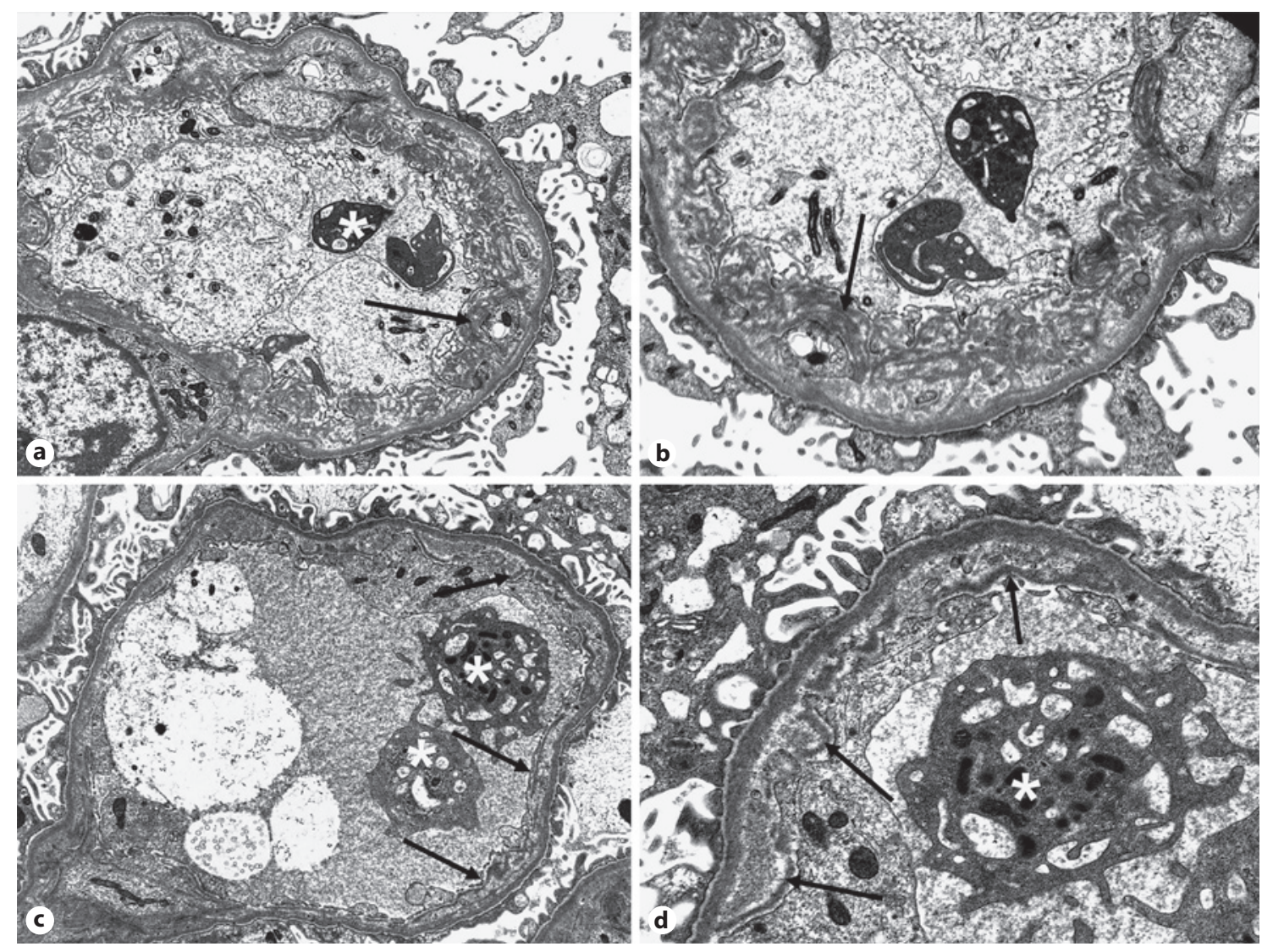

Fig. 11. Renal allograft biopsy at 2 years post-transplant with recurrent atypical hemolytic uremic syndrome, negative for DSA. Glomerular capillaries showing severe TG with intraluminal platelet accumulation $\left({ }^{*}\right)$, severe endothelial cell swelling (a, b), subendothelial widening, multiple GBM reduplications (arrows), and patchy glomerular epithelial cell foot process effacement. $\mathbf{a} \times 10,000$. $\mathbf{b} \times 15,000$. $\mathbf{c} \times 10,000$. $\mathbf{d} \times 20,000$. TG, transplant glomerulopathy; GBM, glomerular basement membrane; DSA, donor-specific antibody.

by endothelial and/or mesangial cell processes within the expanded subendothelial space and in between the reduplicated new GBM layer and the pre-existing GBM can be seen, especially in advanced disease stages (Fig. 8, 9). Occasionally, this cellular interposition may be almost circumferential (Fig. 12c, d). The expanded subendothelial space contains variable heterogeneous nonspecific electron dense flocculent or fibrillar material (Fig. 6a, 7d, $10 \mathrm{c})$.

Typical immune complex-type deposits are usually not present although scattered segmental subendothelial and mesangial deposits have been reported in a subset of human TG biopsies after careful exclusion of recurrent immune complex-mediated glomerulonephritis and hepatitis Cinfection [18]. With further progression of the lesion, multiple layers of subendothelial reduplicated
GBM are being formed, producing a complex woven meshwork of basement membrane/densa-like material (Fig. 9b, 10d). Other ultrastructural glomerular changes in TG are mesangial matrix expansion, occasional endothelial tubuloreticular inclusions [18], and variable podocyte foot process effacement (Fig. 9, 12).

Associated with the development of TG, peritubular capillaries show similar endothelial and basement membrane alterations [35] although those may be lacking in early cases of TG [36]. When present, there is peritubular capillary endothelial cell swelling with loss of fenestrations, and progressive basement membrane multilayering, with 5 or more layers in at least 3 capillaries, and $\geq 7$ layers in at least 1 capillary, considered significant for chronic ABMR [36]. 


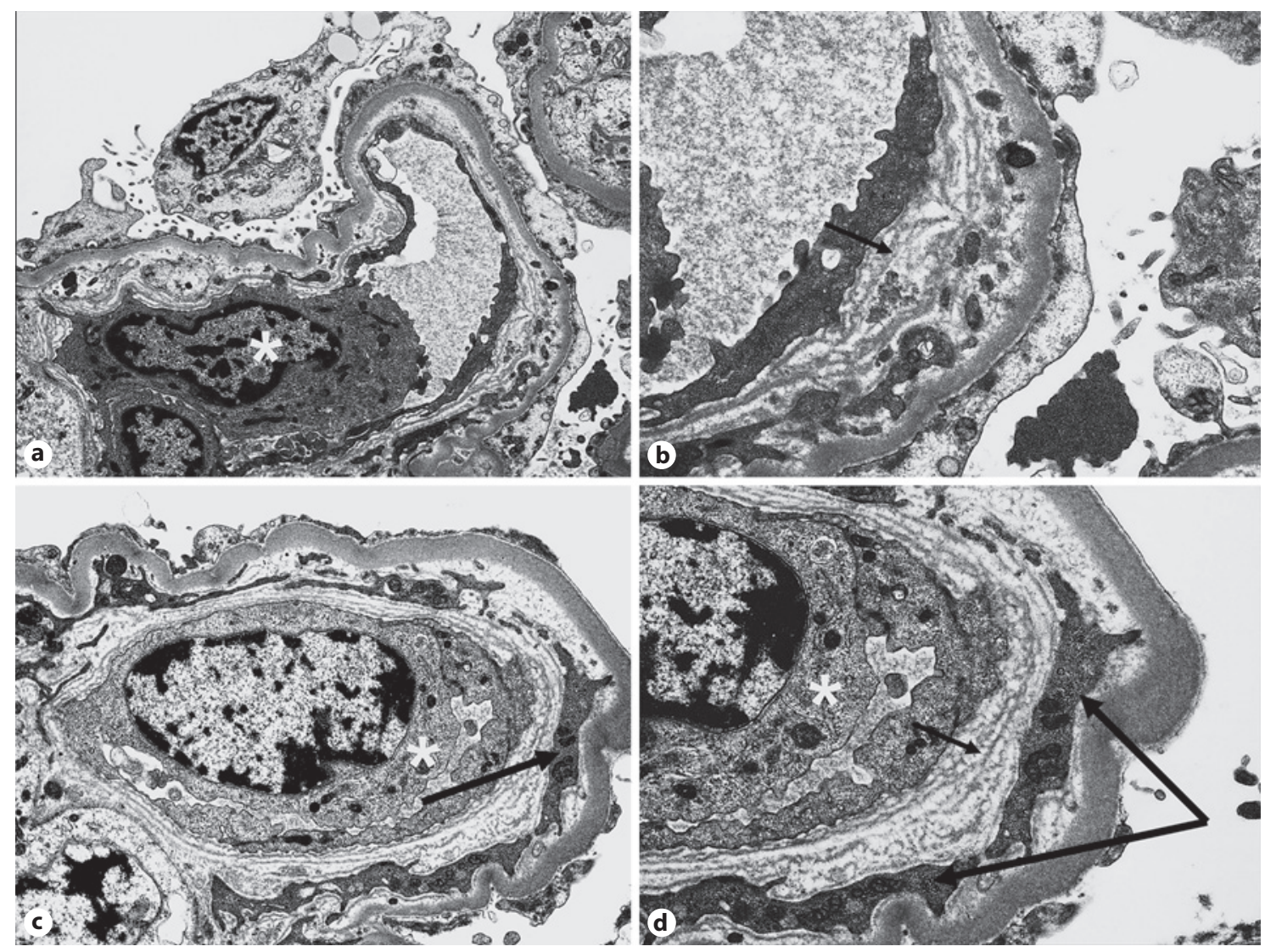

Fig. 12. Renal allograft biopsy at 3 years post-transplant with severe TG (Banff score cg3), in a patient positive for HCV, negative for DSA. Allograft failure occurred 2 years after this biopsy at 5 years post-transplant. Glomerular capillaries showing intracapillary mononuclear cells $\left(\mathbf{a}^{*}\right)$, severe endothelial cell swelling $\left(\mathbf{c}, \mathbf{d}^{*}\right)$, subendothelial widening, multiple circumferential GBM reduplications (b, $\mathbf{d}$ small arrows), almost circumferential mesangial cellular interposition in the subendothelial space (c, $\mathbf{d}$ large arrows), and severe glomerular epithelial cell foot process effacement. $\mathbf{a} \times 10,000$. b $\times 15,000$. $\mathbf{c} \times 10,000$. d $\times 15,000$. TG, transplant glomerulopathy; GBM, glomerular basement membrane; DSA, donor-specific antibody.

\section{Differential Diagnosis of Transplant Glomerulopathy}

GBM reduplications can also be seen in other glomerular diseases such as immune complex-mediated glomerulonephritis. As a general rule TG is only diagnosed in the confirmed absence of a proliferative/immune complexmediated glomerular lesion; a diagnosis that is facilitated by accompanying "negative" IF and electron microscopy (EM) studies. Chronic thrombotic microangiopathy associated with drug toxicity, in particular calcineurin inhibitor toxicity, may cause a similar morphological pattern of glomerular injury. Recurrence of atypical hemolytic uremic syndrome gives essentially identical glomerular morphologic changes to TG (Fig. 11), as does an underlying chronic HCV infection (Fig. 12). In many cases detailed clinicopathological correlations can resolve most diagnostic uncertainties.

\section{Banff Grading of Transplant Glomerulopathy}

The lesion of TG is one of the chronic Banff scores (cg score) in the Banff classification for renal allograft pathology $[37,38]$. The severity of TG is graded according to the percentage of capillary loops showing GBM reduplications in the most severely affected glomerulus [39]. Detailed grading of TG requires both LM and EM studies. Banff score cg0 indicates no evidence of GBM reduplications by LM or EM. If early morphological features of TG are identified only by EM, with no definite GBM redupli- 
cations seen on LM, then Banff score cgla is assigned (Fig. 3, 4). Banff cglb indicates up to $25 \%$ of capillary loops showing reduplications identified by LM (Fig. 1a, b), cg2 indicates $26-50 \%$ of capillary loops showing reduplications, and cg3 indicates severe TG with $>50 \%$ of capillary loops showing reduplications (Fig. 1c, d).

\section{Recommended Glomerular Electron Microscopy Sampling of Renal Allograft Biopsies}

In the past, sampling of renal allograft biopsy tissue for glomerular EM was usually based on clinical suspicion of either recurrent or de novo glomerular disease, such as the presence of proteinuria or hematuria. This approach precludes the opportunity of detecting early or subclinical glomerular disease, which may be more amenable to therapeutic intervention. Employing routine EM examination helps in this regard [40, 41]. The early ultrastructural changes of TG are relatively specific for $\mathrm{ABMR}$ and are found in both C4d-positive and in C4d-negative diagnostic biopsies [26]. The finding of ultrastructural changes of early TG in a renal allograft biopsy should initiate careful investigation for circulating DSA, including both HLA and non-HLA antibodies.

In view of the importance of ultrastructural examination, the Banff Classification now recommends performing $\mathrm{EM}$ on all biopsies from patients at immunological high risk of developing ABMR, including those who are pre-sensitized with DSA, those who develop de novo DSA at any time period post-transplant, and those who have had peritubular capillary C4d deposition, glomerulitis, or peritubular capillaritis in preceding biopsies [34]. It is also suggested that EM be performed routinely on all late indication renal allograft biopsies performed beyond 1 year post-transplantation. At least 10 glomerular capillary loops from at least one nonsclerotic glomerulus per biopsy should be examined ultrastructurally to search for TG. All available functional glomeruli for electron microscopic examination should be examined, as TG changes are often focal, and can show variable severity between glomeruli. In addition, it is recommended that at least 10 peritubular capillaries from areas with most pronounced changes are ultrastructurally examined in a targeted manner for severity of basement membrane multilayering [36].

If early TG ultrastructural changes are identified (Banff cg1a), this should prompt more rigorous testing for DSA. If DSA is identified, then early therapeutic interventions may be beneficial in preventing or slowing the development of TG and progressive allograft dysfunction.

\section{Conclusion}

TG is a manifestation of chronic sustained endothelial injury in renal allografts and represents a morphologic pattern - not necessarily indicating a specific diagnosis. Several diseases and underlying etiologies may result in the phenotype of TG. In kidney transplant recipients, endothelial cell injury and TG is often due to repetitive or persistent/chronic antibody-induced injury and used as one sign of chronic ABMR. Early TG lesions noted by EM may be reversible with aggressive targeted antirejection therapy. Once fully developed, TG is progressive and irreversible, with a poor renal allograft prognosis. An early diagnosis of TG established by routine ultrastructural examination is beneficial for patient management, in particular in patients at high risk for ABMR.

\section{Acknowledgement}

Thanks to Dr. V. Nickeleit and Dr. S. Singh for giving helpful contributions to the manuscript. Thanks also to Mr. G. Burgess and Mr. A. Pobre for technical expertise in EM, and to Dr. C. Wiebe for providing clinical information relevant to some of the allograft biopsies illustrated in this manuscript.

\section{Conflict of Interest Statement}

The author has no conflicts of interest to declare.

\section{Funding Sources}

No relevant funding source to declare.

\section{Author Contributions}

I.W.G. wrote this paper.

\section{References}

1 Sis B, Campbell PM, Mueller T, Hunter C, Cockfield SM, Cruz J, et al. Transplant glomerulopathy, late antibody-mediated rejection and the ABCD tetrad in kidney allograft biopsies for cause. Am J Transplant. 2007; 7(7):1743-52.

2 Yang Y, Hodgin JB, Afshinnia F, Wang SQ, Wickman L, Chowdhury M, et al. The two kidney to one kidney transition and transplant glomerulopathy: a podocyte perspective. J Am Soc Nephrol. 2015;26(6):145065. 
3 Sis B, Jhangri GS, Bunnag S, Allanach K, Kaplan B, Halloran PF. Endothelial gene expression in kidney transplants with alloantibody indicates antibody-mediated damage despite lack of C4d staining. Am J Transplant. 2009; 9(10):2312-23.

4 Lubetzky M, Hayde N, Ó Broin PP, Ajaimy M, Bao Y, Mohammed O, et al. Molecular signatures and clinical outcomes of transplant glomerulopathy stratified by microvascular inflammation and donor-specific antibody. Clin Transplant. 2019;33(3):e13469.

5 Bagnasco SM, Zachary AA, Racusen LC, Arend LJ, Carter-Monroe N, Alachkar N, et al. Time course of pathologic changes in kidney allografts of positive crossmatch HLA-incompatible transplant recipients. Transplantation. 2014;97(4):440-5.

6 Wiebe C, Gibson IW, Blydt-Hansen TD, Pochinco D, Birk PE, Ho J, et al. Rates and determinants of progression to graft failure in kidney allograft recipients with de novo donorspecific antibody. Am J Transplant. 2015; 15(11):2921-30.

7 Gloor JM, Cosio FG, Rea DJ, Wadei HM, Winters JL, Moore SB, et al. Histologic findings one year after positive crossmatch or $\mathrm{ABO}$ blood group incompatible living donor kidney transplantation. Am J Transplant. 2006;6(8):1841-7.

8 Dinavahi R, George A, Tretin A, Akalin E, Ames S, Bromberg JS, et al. Antibodies reactive to non-HLA antigens in transplant glomerulopathy. J Am Soc Nephrol. 2011;22(6): 1168-78.

9 Joosten SA, Sijpkens YW, van Ham V, Trouw LA, van der Vlag J, van den Heuvel B, et al. Antibody response against the glomerular basement membrane protein agrin in patients with transplant glomerulopathy. Am J Transplant. 2005;5(2):383-93.

10 Gunasekaran M, Maw TT, Santos RD, Shenoy $S$, Wellen J, Mohanakumar T. Immunoglobulin isotype switching of antibodies to vimentin is associated with development of transplant glomerulopathy following human renal transplantation. Transpl Immunol. 2017;45: 42-7.

11 Haas M. The relationship between pathologic lesions of active and chronic antibody-mediated rejection in renal allografts. Am J Transplant. 2018;18(12):2849-56.

12 Smith RN, Kawai T, Boskovic S, Nadazdin O, Sachs DH, Cosimi AB, et al. Four stages and lack of stable accommodation in chronic alloantibody-mediated renal allograft rejection in Cynomolgus monkeys. Am J Transplant. 2008;8(8):1662-72.

13 Wiebe C, Gibson IW, Blydt-Hansen TD, Karpinski M, Ho J, Storsley LJ, et al. Evolution and clinical pathologic correlations of de novo donor-specific HLA antibody post kidney transplant. Am J Transplant. 2012;12(5): 1157-67.
14 Gallay BJ, Alpers CE, Davis CL, Schultz MF, Johnson RJ. Glomerulonephritis in renal allografts associated with hepatitis $\mathrm{C}$ infection: a possible relationship with transplant glomerulopathy in two cases. Am J Kidney Dis. 1995;26(4):662-7.

15 Baid S, Pascual M, Williams WW Jr, TolkoffRubin N, Johnson SM, Collins B, et al. Renal thrombotic microangiopathy associated with anticardiolipin antibodies in hepatitis C-positive renal allograft recipients. J Am Soc Nephrol. 1999;10(1):146-53.

16 Baid-Agrawal S, Farris AB, Pascual M, Mauiyyedi S, Farrell ML, Tolkoff-Rubin N, et al. Overlapping pathways to transplant glomerulopathy: chronic humoral rejection, hepatitis $\mathrm{C}$ infection, and thrombotic microangiopathy. Kidney Int. 2011;80(8):879-85.

17 Cosio FG, Roche Z, Agarwal A, Falkenhain ME, Sedmak DD, Ferguson RM. Prevalence of hepatitis $\mathrm{C}$ in patients with idiopathic glomerulopathies in native and transplant kidneys. Am J Kidney Dis. 1996;28(5):752-8.

18 Grau V, Zeuschner P, Immenschuh S, Bockmeyer CL, Zell S, Wittig J, et al. Immune complex-type deposits in the Fischer-344 to Lewis rat model of renal transplantation and a subset of human transplant glomerulopathy. Transplantation. 2016;100(5):1004-14.

19 Adam BA, Smith RN, Rosales IA, Matsunami M, Afzali B, Oura T, et al. Chronic antibodymediated rejection in nonhuman primate renal allografts: validation of human histological and molecular phenotypes. Am J Transplant. 2017;17(11):2841-50.

20 Gloor JM, Sethi S, Stegall MD, Park WD, Moore SB, DeGoey S, et al. Transplant glomerulopathy: subclinical incidence and association with alloantibody. Am J Transplant. 2007;7(9):2124-32

21 Cosio FG, Gloor JM, Sethi S, Stegall MD. Transplant glomerulopathy. Am J Transplant. 2008;8(3):492-6.

22 Kieran N, Wang X, Perkins J, Davis C, Kendrick E, Bakthavatsalam R, et al. Combination of peritubular c4d and transplant glomerulopathy predicts late renal allograft failure. J Am Soc Nephrol. 2009;20(10):2260-8.

23 Aubert O, Higgins S, Bouatou Y, Yoo D, Raynaud M, Viglietti D, et al. Archetype analysis identifies distinct profiles in renal transplant recipients with transplant glomerulopathy associated with allograft survival. J Am Soc Nephrol. 2019;30(4):625-39.

24 Kovacs G, Devercelli G, Zelei T, Hirji I, Vokó Z, Keown PA. Association between transplant glomerulopathy and graft outcomes following kidney transplantation: a meta-analysis. PLoS One. 2020;15:e231646.

25 Moreso F, Crespo M, Ruiz JC, Torres A, Gutierrez-Dalmau A, Osuna A, et al. Treatment of chronic antibody mediated rejection with intravenous immunoglobulins and rituximab: a multicenter, prospective, randomized, double-blind clinical trial. Am J Transplant. 2018; 18(4):927-35.
26 Haas M, Mirocha J. Early ultrastructural changes in renal allografts: correlation with antibody-mediated rejection and transplant glomerulopathy. Am J Transplant. 2011; 11(10):2123-31.

27 Kahwaji J, Najjar R, Kancherla D, Villicana R, Peng A, Jordan S, et al. Histopathologic features of transplant glomerulopathy associated with response to therapy with intravenous immune globulin and rituximab. Clin Transplant. 2014;28(5):546-53.

28 Choi J, Aubert O, Vo A, Loupy A, Haas M, Puliyanda D, et al. Assessment of tocilizumab (anti-interleukin-6 receptor monoclonal) as a potential treatment for chronic antibody-mediated rejection and transplant glomerulopathy in HLA-sensitized renal allograft recipients. Am J Transplant. 2017;17(9):2381-9.

29 Lavacca A, Presta R, Gai C, Mella A, Gallo E, Camussi G, et al. Early effects of first-line treatment with anti-interleukin-6 receptor antibody tocilizumab for chronic active antibody-mediated rejection in kidney transplantation. Clin Transplant. 2020;34(8):e13908.

30 Panzer SE, Joachim E, Parajuli S, Zhong W, Astor BC, Djamali A. Glomerular C3 deposition is an independent risk factor for allograft failure in kidney transplant recipients with transplant glomerulopathy. Kidney Int Rep. 2019;4(4):582-93.

31 Gasin AH, Chua JS, Wolterbeek R, Schmitz J, Weimer E, Singh HK, et al. Glomerular C4d deposits can mark structural capillary wall remodelling in thrombotic microangiopathy and transplant glomerulopathy: $\mathrm{C} 4 \mathrm{~d}$ beyond active antibody-mediated injury: a retrospective study. Transpl Int. 2017;30:519-32.

32 Roy S, Nalwa A, Keith J, Weck K, Singh H, Nickeleit V. Pseudolinear C4d deposits in a hereditary glomerulopathy caused by a rare NC1 collagen-4-alpha- 5 missense mutation: a "new disease entity"? Ultrastruct Pathol. 2019;43:209-15.

33 Wavamunno MD, O'Connell PJ, Vitalone M, Fung CL, Allen RD, Chapman JR, et al. Transplant glomerulopathy: ultrastructural abnormalities occur early in longitudinal analysis of protocol biopsies. Am J Transplant. 2007; 7(12):2757-68.

34 Haas M, Sis B, Racusen LC, Solez K, Glotz D, Colvin RB, et al. Banff 2013 meeting report: inclusion of C4d-negative antibody-mediated rejection and antibody-associated arterial lesions. Am J Transplant. 2014;14(2):272-83.

35 Monga G, Mazzucco G, Novara R, Reale L. Intertubular capillary changes in kidney allografts: an ultrastructural study in patients with transplant glomerulopathy. Ultrastruct Pathol. 1990;14(3):201-9.

36 Liapis G, Singh HK, Derebail VK, Gasim AM, Kozlowski T, Nickeleit V. Diagnostic significance of peritubular capillary basement membrane multilaminations in kidney allografts: old concepts revisited. Transplantation. 2012; 94(6):620-9. 
37 Haas M, Loupy A, Lefaucheur C, Roufosse C, Glotz D, Seron D, et al. The Banff 2017 kidney meeting report: revised diagnostic criteria for chronic active $\mathrm{T}$ cell-mediated rejection, antibody-mediated rejection and prospects for integrative endpoints for next-generation clinical trials. Am J Transplant. 2018;18(2): 293-307.
38 Loupy A, Haas M, Roufosse C, Naesens M, Adam B, Afrouzian M, et al. The Banff 2019 kidney meeting report (I): updates on and clarification of criteria for $\mathrm{T}$ cell- and antibody-mediated rejection. Am J Transplant. 2020 May 28;20:2318-31. online ahead of print.

39 Roufosse C, Simmonds N, Clahsen-van Gronigen $\mathrm{M}$, Haas M, Henriksen KJ, Horsfield C, et al. A 2018 reference guide to the Banff classification of renal allograft pathology. Transplantation. 2018;102:1795-814.
40 Ivanyi B. Transplant capillaropathy and transplant glomerulopathy: ultrastructural markers of chronic renal allograft rejection. Nephrol Dial Transplant. 2003;18(4):655-60.

41 deKort H, Moran L, Roufosse C. The role of electron microscopy in renal allograft biopsy evaluation. Curr Opin Organ Transplant. 2015;20:333-42. 